\title{
HOMEOMORPHISM GROUPS AND COSET SPACES $\left({ }^{1}\right)$
}

\author{
BY
}

LESTER R. FORD, JR.

1. Introduction. This paper considers the problem of giving a topology to a homeomorphism group. This problem was considered by Arens [1] $\left.{ }^{2}\right)$ for a group of homeomorphisms of a locally compact space. The present paper attempts to obtain similar results for arbitrary completely regular spaces. All spaces hereafter will be taken as Hausdorff spaces.

The numbers here refer to sections. In (2) we give a (usually) nontrivial topology for an arbitrary homeomorphism group of any completely regular space. Since the convergence notion used is that of uniform convergence, the topology is easily seen to be admissible in the sense of Arens [1]. In (3) we restrict our considerations to transitive homeomorphism groups. We define a "reasonable" topology for the group to be one which makes the space homeomorphic to the space of cosets of the group modulo that subgroup leaving some particular element fixed. We develop some of the properties of reasonability.

In (4) we present a sufficient condition (Definition 4.1) for a space to possess a homeomorphism group with a reasonable topology, and show that certain well known spaces, in particular the manifolds, possess this property. In (5) we give two examples. The first shows that the sufficient condition is not a necessary condition. The second shows, however, that some further condition is necessary. This latter example has some interesting connectivity properties in its own right.

2. Homeomorphism groups of completely regular spaces. The main theorem of this section is Theorem 2.2 which makes any group of homeomorphisms of a completely regular space into a topological group under a topology induced by a notion of uniform convergence. This theorem follows rather trivially from the following.

THEOREM 2.1. If $f_{a}, a \in A$, is a directed set of homeomorphisms which are uniformly convergent to some homeomorphism $f$, and if $f^{-1}$ is uniformly continuous, then $f_{a}^{-1}$ converges uniformly to $f^{-1}$.

Proof. We shall denote by German capitals the basic neighborhoods of the diagonal which we shall assume to be symmetric. The proof will be based on the following lemma.

Presented to the Society, December 29, 1953; received by the editors July 26, 1953.

(1) The material of this paper comes from a thesis written under the direction of Professor D. G. Bourgin.

(2) The numbers in brackets refer to the bibliography which appears at the end of this paper. 
Lemma. For every $\mathfrak{U}$ there exists a $\mathfrak{B}$ and an $a(\mathfrak{U})$ such that for $a>a(\mathfrak{U})$ and $(x, y) \in \mathfrak{B}$ we have $\left(f_{a}^{-1} x, f_{a}^{-1} y\right) \in \mathfrak{U}$.

Proof. By the uniform continuity of $f^{-1}$ there exists, for each $\mathfrak{U}$, a $\mathfrak{W}$ such that $(x, y) \in \mathfrak{W}$ implies that $\left(f^{-1} x, f^{-1} y\right) \in \mathfrak{U}$. Take now $\mathfrak{B}^{3} \subset \mathfrak{W}$ and take $a(\mathfrak{U})$ large enough so that $a>a(\mathfrak{U})$ implies that $\left(f_{a} x, f x\right) \in \mathfrak{B}$ for all $x$. Now notice that $(x, y) \in \mathfrak{B}$ implies that $\left(f_{a} f_{a}^{-1} x, f_{a} f_{a}^{-1} y\right) \in \mathfrak{B}$. But $\left(f f_{a}^{-1} y, f_{a} f_{a}^{-1} y\right)$ and $\left(f_{a} f_{a}^{-1} x, f f_{a}^{-1} x\right)$ are both in $\mathfrak{B}$ and thus $\left(f f_{a}^{-1} x, f f_{a}^{-1} y\right) \in \mathfrak{W}$. Therefore $\left(f_{a}^{-1} x, f_{a}^{-1} y\right)$ $\in \mathfrak{U}$ by an application of $f^{-1}$, and the lemma is proved.

To complete the proof of the theorem let $\mathfrak{U}$ be given. Find $\mathfrak{B}$ and $a_{0}$ as in the lemma. Let $a_{1}$ be such that, for $a>a_{1},\left(f_{a} x, f x\right) \in \mathfrak{B}$. Let $a_{2}$ be larger than both $a_{0}$ and $a_{1}$. Then for $a>a_{2}$ and arbitrary $z=f x$ we have $\left(f_{a} x, f x\right) \in \mathfrak{B}$. Thus $\left(x, f_{a}^{-1} f x\right) \in \mathfrak{U}$ from the lemma, and this pair is precisely $\left(f^{-1} z, f_{a}^{-1} z\right)$. Thus the convergence is uniform.

Using the above theorem we have immediately the following theorem.

THEOREM 2.2. If $X$ possesses a uniform structure under which every element of some homeomorphism group $G$ is uniformly continuous, then $G$ is a topological group relative to the uniform convergence notion induced by the uniformity.

If $G$ is a homeomorphism group and $x$ is a point in the space, we denote by $C_{x}$ the subgroup of $G$ having $x$ as a fixed point. It is well known that there is a natural mapping $\eta: G / C_{x} \rightarrow O(x)$ where $O(x)$ is the orbit of $x$ under $G$. One easily sees that $\eta$ is $1-1$ and is continuous whenever the $G$ topology is finer than the point open topology (in particular, if the $G$ topology is generated by a uniform structure on $X$ ). We might also remark that the uniform topology on $G$ is admissible in the sense of Arens.

3. Reasonable homeomorphism groups. We shall now define, for the special case in which the group is transitive, a "reasonable" topology. Let $G$ be transitive on $X, C_{x}$ defined as above, then:

Definition 3.1. $G$ has a reasonable topology over $X$ if $G$ is a topological group under this topology, and the coset space $G / C_{x}$ (left cosets for definiteness) is homeomorphic to $X$ under the map $\eta: G / C_{x} \rightarrow X$ defined by $\eta\left(g C_{x}\right)$ $=g(x)$.

An immediate result of this is that $C_{x}$ is a closed subgroup of $G$ since $X$ is a space in which points are closed. Furthermore $X$ is Hausdorff since a topological group manages to pass this property along to its coset spaces. The following theorem shows that $X$ is also completely regular. I present it in detail because I shall need the explicit construction of the uniform structure in order to prove several useful corollaries.

THEOREM 3.1. If $G$ is a topological group and $F$ a closed subgroup of $G$, then $G / F$ possesses a uniform structure agreeing with its topology and induced naturally by the uniform structure on $G$. 
Proof. Let $\mathfrak{F}=\left\{U^{a}(e)\right\}$ be a basic system of symmetric neighborhoods of the identity in $G$. These are easily seen to generate a uniformity when we define $U^{a}(\Delta)$ as a neighborhoood of the diagonal by $\left(g_{1}, g_{2}\right) \in U^{a}(\Delta)$ if and only if $g_{1} \in U^{a}(e) \cdot g_{2}$. Further let $\phi: G \rightarrow G / F$ be the canonical mapping and define $\phi^{*}: G \times G \rightarrow G / F \times G / F$ by $\phi^{*}\left(g_{1}, g_{2}\right)=\left[\phi\left(g_{1}\right), \phi\left(g_{2}\right)\right]$. We shall use as a basis for our uniformity on $G / F$ all sets of the form $\phi^{*}\left[U^{a}(\Delta)\right]$.

That these neighborhoods contain the diagonal and are symmetric is trivial. Furthermore, if $V \subset U \cap W$ in the $G$ uniformity then $\phi^{*}(V)$ $\subset \phi^{*}(U \cap W) \subset \phi^{*}(U) \cap \phi^{*}(W)$. All that remains to be established is the "square root" property. For this we need only show that if $(u, v)$ and $(x, y)$ are in $W$ where $W^{2} \subset V$ and if $\phi(v)=\phi(x)$, then $[\phi(u), \phi(y)] \in \phi^{*}(V)$. However if $u \in W v$ then $u F \subset W v F=W x F$. Furthermore, if we assume that $W x \cap u F=\varnothing$, then $W x f \cap u F=\varnothing$ for every $f$ in $F$. Hence $W x F \cap u F=\varnothing$ contradicting the above. Similarly we can show that $W x \cap y F \neq \varnothing$, and we may choose $u^{\prime}$ and $y^{\prime}$ in $W x \cap u F$ and $W x \cap y F$ respectively. The remainder is trivial.

In a similar fashion we easily show that this uniform structure actually agrees with the topology on $G / F$.

COROLlARY 1. If $G$ is a reasonable group of homeomorphisms of $X$, then $X$ possesses a uniform structure and is therefore completely regular.

COROLLARY 2. The convergence of directed systems in $G$ implies the uniform convergence of those same directed systems relative to the induced uniformity as described in the theorem. (Here elements of $G$ are treated as homeomorphisms of $G / F$.)

CoROllary 3. Every $g$ in $G$, considered as an operator on $G / F$, is uniformly continuous with respect to the induced uniform structure.

The proofs of the above corollaries are rather trivial and will be omitted. As an example of a space which possesses a transitive homeomorphism group with no reasonable topology we take the plane. Let basic neighborhoods of the point $\left(x_{0}, y_{0}\right)$ be all sets of the form:

$$
N_{e}\left(x_{0}, y_{0}\right)=\left[(x, y) \mid x_{0}<x<x_{0}+e ; y_{0}-e<y<y_{0}+e\right] \cup\left(x_{0}, y_{0}\right) .
$$

The Euclidean translations are evidently homeomorphisms so that $X$ is homogeneous. One easily sees that $X$ is not completely regular. In fact $X$ is not even regular since the point $(0,0)$ and the closed set $[(x, y) \mid x=0 ; y \neq 0]$ can not be separated by open sets.

Theorem 3.2. Suppose $T_{1}$ and $T_{2}$ are topologies on $G$ such that $G\left(T_{1}\right)$ is finer than $G\left(T_{2}\right)$ which is in turn finer than the point-open topology. Then if $G\left(T_{1}\right)$ is reasonable over $X, G\left(T_{2}\right)$ must also be reasonable over $X$.

Proof. The continuity of the identity map from $G\left(T_{1}\right)$ to $G\left(T_{2}\right)$ induces a 
continuous identity map between the corresponding coset spaces. But the continuity of the inverse is merely the continuity of $\eta$ which has been previously established.

We shall further note for later use that for fixed $x_{0}$ the map $\pi$ of $G$ in the point-open topology onto $X$ defined by $\pi(g)=g\left(x_{0}\right)$ must be open if $G$ possesses a reasonable topology over $X$. We may now state the following theorem which enables us to restrict our search for a reasonable topology to those topologies which are generated by a notion of uniform convergence.

THEOREM 3.3. If $G$ is reasonable over $X$, then there exists a uniform structure agreeing with the topology of $X$ so that if $G$ is given this uniform topology, then $G$ is still reasonable over $X$.

Proof. This follows immediately from Corollaries 2 and 3 of 3.1, and Theorems 2.2 and 3.2 .

4. Strong local homogeneity. We have seen several necessary conditions for $G$ to be reasonable over $X$, but as yet no sufficient conditions. I propose the following sufficient condition. In addition I think that it has some interest in its own right.

Definition 4.1. A space $X$ is S.L.H. (strong local homogeneity) if for every neighborhood of any point $x$, there exists a subneighborhood $U(x)$ such that for any $z \in U(x)$ there exists a homeomorphism $g$ with $g(x)=z$ and with $g$ equal to the identity on the complement of $U(x)$.

We shall immediately prove the theorem which justifies this definition.

THEOREM 4.1. Suppose $G$ is the full homeomorphism group of an S.L.H., completely regular space $X$, and suppose $G$ is transitive. Then $G$ is reasonable over $X$ under any topology induced by a uniform structure on $X$.

Proof. $\eta: G / C_{x} \rightarrow X$ is already known to be continuous, and 1-1. Hence we need only show openness. For $U(e)$ any neighborhood of the identity we let, in $X, V^{2} \subset U$ and let $N$ be an S.L.H. neighborhood of $x$ contained in $V(x)$. We shall see that $U(e)$ maps onto $N$. For if $z \in N$, then there exists $g$ as in the definition of S.L.H. taking $x$ into $z$. But then for $y$ in the complement of $N$ we clearly have $[g(y), y] \in U$, and for $y \in N$ we have $[g(y), x] \in V$ and $[x, y]$ $\in V$ which implies that $[g(y), y] \in U$. Thus $g \in U(e)$ and $\eta$ is open for neighborhoods of the identity. Therefore $\eta$ is open for arbitrary neighborhoods.

We shall now examine certain spaces and show that they are completely regular, S.L.H., and homogeneous, which will imply that they possess reasonable homeomorphism groups and can be represented as coset spaces of some topological group.

THEOREM 4.2. If $X$ is homogeneous and has a basis for its topology composed of sets which are both open and closed, then $X$ is S.L.H. and completely regular.

Proof. Complete regularity is immediate since the characteristic function 
of any one of the basic sets must be continuous.

For $U(x)$ an arbitrary neighborhood of $x$ we let $V(x) \subset U(x)$ be both open and closed. For any $z \in V(x)$ there exists $W_{1}(x)$ and $W_{2}(z)$ contained in $V$, disjoint, and each both open and closed. Let $h$ be a homeomorphism taking $x$ into $z$ and let $Z=W_{1} \cap h^{-1}\left(W_{2}\right) . Z$ and $h(Z)$ are disjoint, and each is both open and closed. Define $\phi$ by:

$$
\begin{aligned}
& \phi(y)=h(y) \text { for } y \in Z \\
& \phi(y)=h^{-1}(y) \text { for } y \in h(Z) \\
& \phi(y)=y \text { for all other } y \text { in the space. }
\end{aligned}
$$

$\phi$ is clearly a homeomorphism and is also the identity on the complement of $V$ since $Z \cup h(Z) \subset V$.

ThEOREM 4.3. If $X$ is a normed linear space, then $X$ is S.L.H., completely regular, and homogeneous.

Proof. Homogeneity and complete regularity follow immediately. We shall establish S.L.H. for the unit sphere only, considered as a neighborhood of the zero, since translations and scalar multiplication will do the rest. Let $x_{0}$ have norm less than 1 and nonzero. Consider the transformation $T(x)$ defined by:

$$
T(x)=\left\{\begin{array}{ccc}
x+x_{0}(1-\|x\|) & \text { for } & \|x\| \leqq 1 \\
x & \text { for } & \|x\| \geqq 1 .
\end{array}\right.
$$

We easily see that the following inequalities hold.

$$
\left(1+\left\|x_{0}\right\|\right)\|x-y\| \geqq\|T(x)-T(y)\| \geqq\left(1-\left\|x_{0}\right\|\right)\|x-y\| .
$$

These immediately imply that $T$ is a homeomorphism, and one sees also that $T$ is the identity on the boundary and exterior of the unit sphere, and takes the zero into $x_{0}$.

Corollary 1. If $X$ is locally Euclidean and Hausdorff, then $X$ is S.L.H.

COROLLARY 2. Every manifold possesses a reasonable homeomorphism group; i.e., every manifold may be represented as a coset space of some topological group by some suitable subgroup.

5. Two examples. Our first example will show that S.L.H. is much too strong a requirement. We shall give a space which is homogeneous, completely regular, and with a reasonable homeomorphism group, but which is not S.L.H.

In particular let $C$ be the Cantor set and let $S^{1}$ be the 1-dimensional circle. The Cartesian product of two homogeneous spaces is also homogeneous, and these spaces are easily seen to be homogeneous. Furthermore the product space is compact and hence is completely regular and possesses a unique 
uniform structure. We shall therefore assume it to be imbedded on the surface of a right circular cylinder in Euclidean 3-space with the metric topology. The space is evidently not S.L.H. since in order to move $x$ to a neighboring point on a different circle requires moving the whole circle. But it is easily seen that for $d(x, z)<e$ there exists a homeomorphism $g$ taking $x$ into $z$ which is uniformly within $e$ of the identity. Hence the full homeomorphism group of $C \times S^{1}$ possesses a reasonable topology.

The second example is not quite so simple. It is an example of a space which is completely regular and homogeneous with the property that no transitive homeomorphism group may be given a reasonable topology.

Definition 5.1. Let $\left\{a_{n}\right\}, n=0, \pm 1, \pm 2, \cdots$, be the class of all sequences of real numbers such that for each sequence there is an index $n_{0}$ so that for $n>n_{0}$ we have $a_{n}=0$ and for $n \leqq n_{0}$ we have $a_{n}>0$. We shall topologize our space by prescribing $e$-neighborhoods of the points, for $e>0$, as follows: $b_{n} \in N_{e}\left(a_{n}\right)$ if and only if :

(1) For the $n_{0}$ associated with $a_{n}$ we have $a_{n}=b_{n}$ for all $n<n_{0}$.

(2) $\left(\sum\left(a_{n}-b_{n}\right)^{2}\right)^{1 / 2}<e$.

We should remark at this point that the map $\phi_{k}: X \rightarrow X$ defined by $\phi_{k}\left(a_{n}\right)=b_{n}$ where $b_{n}=a_{n+k}$ is a homeomorphism. The fact that we do have a topology is a trivial consequence of the Schwartz inequality.

Part I. Homogeneity. Let $C\left[a_{n}\right]$ be the class of all points which ultimately agree with $a_{n}$ for increasingly large negative indices. One sees immediately that $C\left[a_{n}\right]$ is both open and closed. Now suppose that $a_{n}$ and $b_{n}$ are chosen and we shall construct a homeomorphism from $C\left[a_{n}\right]$ onto $C\left[b_{n}\right]$ taking $a_{n}$ onto $b_{n}$. By using a suitable $\phi_{k}$ we may clearly assume that the $n_{0}$ of the definition is the same for each, say equal to zero. Let $h_{0}, h_{-1}, h_{-2}, \cdots$ be a collection of homeomorphisms of the positive real line onto itself, chosen so that $h_{-n}\left(a_{-n}\right)=b_{-n}$. Let $H: C\left[a_{n}\right] \rightarrow C\left[b_{n}\right]$ be the composite map defined by letting $h_{-n}$ operate on the $-n$th coordinate and using the identity map on those coordinates with positive indices. $H$ is evidently $1-1$ onto. Further since $H^{-1}$ is another map of the same type it suffices to show continuity in one direction. But if $N_{e}\left(c_{n}\right)$ is an arbitrary neighborhood then, if the associated $n_{0}$ is larger than zero the image is merely another neighborhood of the same type, and if less than zero, only those coordinates between $n_{0}$ and zero get altered in such a manner as to affect the neighborhood as defined. Since there are only a finite number of them we have essentially a homeomorphism of a finitedimensional Euclidean space defined by coordinatewise homeomorphisms. Hence $H$ is the desired homeomorphism.

Part II. Complete regularity. Inside $U\left(a_{n}\right)$ we find $N_{e}\left(a_{n}\right)$ and define a function by:

$$
f\left(b_{n}\right)=\frac{\left(\sum\left(a_{n}-b_{n}\right)^{2}\right)^{1 / 2}}{e}
$$$$
\text { for } b_{n} \in N_{e} \text {, }
$$ 


$$
f\left(b_{n}\right)=1
$$

The continuity of $f$ is easily established by considering inverse images of subbasic sets on the unit interval.

Part III. We shall now show that if we give $G$ the point open topology, then the map $\pi$ defined at the end of $\S 3$ is not an open map. We shall need a few lemmas.

LEMмA 1. If $\left[\cdots, a_{-1}, a_{0}, 0,0, \cdots\right]$ is removed from the space, then the following sets are open, disjoint, and exhaust the space.

$$
\begin{aligned}
& S_{1}=\left[b_{n} \mid b_{n}=a_{n} \text { for } n=0,-1,-2, \cdots\right], \\
& S_{2}=\left[b_{n} \mid b_{n}=a_{n} \text { for } n=-1,-2, \cdots ; a_{0}<b_{0}\right], \\
& S_{3}=\text { Remainder of the space. }
\end{aligned}
$$

Proof. This is clear from the definition of $e$-neighborhood and will be omitted, as will the proof of:

Leмma 2. The class of all points of the form $\left[\cdots, a_{-1}, a_{0}, t, 0,0, \cdots\right]$ where the $a_{i}$ are fixed and $0 \leqq t \leqq 2$ is an arc in $X$.

LEMмA 3. $N_{1 / 3}\left[\cdots, a_{0}, 0, \cdots\right], N_{1 / 3}\left[\cdots, a_{0}, 1,0, \cdots\right]$, and $N_{1 / 3}\left[\cdots, a_{0}, 2,0, \cdots\right]$ together with their defining points determine $a$ neighborhood in the point-open topology. Any homeomorphism which is in this neighborhood must take the point $\left[\cdots, a_{0}, 1,0, \cdots\right]$ onto the arc of Lemma 2. (For brevity we shall refer to the above sets as $N(0), N(1)$, and $N(2)$, respectively, and their defining points as 0,1 , and 2.)

Proof. Suppose not. Notice that the point 1 must map into a point of the form $\left[\cdots, a_{0}, 1+k_{1}, k_{2}, \cdots\right]$ where $\left|k_{1}\right|<1 / 3$ and $k_{2} \neq 0$. We shall remove the point $\left[\cdots, a_{0}, 1+k_{1}, 0, \cdots\right]$ from the space. We see that $N(2)$ $\subset S_{2}$ of Lemma 1 , that $N(0) \subset S_{3}$, and that the image of point 1 is contained in $S_{1}$. However, the homeomorphic image of an arc is again an arc, with the transform of point 1 corresponding to some $t$ between zero and one. Since the arc is connected, there must be a $t^{\prime}$ value between 0 and $t$ and a $t^{\prime \prime}$ between $t$ and 1 both of which correspond to the removed point. This contradicts 1-1-ness of the homeomorphism.

We complete the proof of Part III by noting that the image under $\pi_{1}$ of the point-open neighborhood described in Lemma 3 is not an open set, but merely an arc. This completes the counterexample when we apply the necessary condition for reasonability given at the end of $\$ 3$.

This construction in the second example has some interest in its own right. If we restrict our consideration to one of the subspaces $C\left[a_{n}\right]$ we can easily see that it is arcwise connected, locally arcwise connected, and 1-dimensional. However the removal of any single point decomposes the space into precisely three components. One can extend the construction, although 
one has to drop the useful "metric" neighborhoods, so as to get a space with the property that the removal of any single point disjoints it into exactly $n$ distinct components, for quite arbitrary integers $n$.

\section{BIBLIOGRAPHY} 610.

1. R. Arens, Topology for homeomorphism groups, Amer. J. Math. vol. 68 (1946) pp. 593-

2. J. W. Tukey, Convergence and uniformity in topology, Annals of Mathematics Studies, no. 2, Princeton University Press, 1940.

UNIVERSITY OF ILLINOIS,

URBANA, ILL. 